\title{
Fotemustine and recurrent glioblastoma: possible new opportunities for an old drug
}

\author{
Raffaele Addeo $\cdot$ M. Serena De Santi • \\ Salvatore Del Prete $\cdot$ Michele Caraglia
}

Received: 14 June 2009/ Accepted: 14 July 2009/Published online: 29 July 2009

(C) Springer-Verlag 2009

Glioblastoma (glioblastoma multiforme [GBM]) comprises the most common types of primary central nervous system tumors. The median survival of patients with GBMs treated conservatively is 14 weeks; by surgical resection alone, 20 weeks; by surgery and radiation, 36 weeks; and by the addition of chemotherapy, 40-50 weeks [1]. The "gold standard" first-line treatment of GBM is based on the combination between radiotherapy and temozolamide as derived from the study by Stupp et al. [2]. In the latter study, patients were randomized to radiotherapy alone or radiotherapy plus temozolamide. Radiotherapy consisted of fractionated focal irradiation for a total dose of $60 \mathrm{~Gy}$. Concomitant chemotherapy consisted of temozolomide at a dose of $75 \mathrm{mg} / \mathrm{m}^{2}$ per day, given 7 days per week from the first day of radiotherapy until the last day of radiotherapy. After a 4-week break, patients were then to receive up to six cycles of adjuvant temozolomide according to the standard 5-day $150 \mathrm{mg} / \mathrm{m}^{2}$ schedule every 28 days and increased to $200 \mathrm{mg} / \mathrm{m}^{2}$ beginning with the second cycle. The hazard ratio (HR) for death among patients treated with radiotherapy plus temozolomide, when compared with those who received radiotherapy alone, was 0.63 (95\%

R. Addeo $(\bowtie) \cdot$ S. Del Prete

Oncology Unit, Oncology Department, "S. Giovanni di Dio"

Hospital, ASL Napoli 2 Nord, Via Giovanni XXIII,

Frattaminore, 80028 Naples, Italy

e-mail: lelloaddeo@alice.it

M. S. De Santi

Neurosurgery Unit, "Umberto I" Hospital, Nocera Inferiore,

Salerno, Italy

M. Caraglia $(\bowtie)$

Department of Biochemistry and Biophysics, Second University

of Naples, Via Costantinopoli, 16, 80138 Naples, Italy

e-mail: michele.caraglia@unina2.it confidence interval, $0.52-0.75 ; P \leq 0.001)$ thus indicating the use of this combination for the treatment of GBM [2]. The 5-year survey of this study confirmed an advantage for combined therapy with an overall survival (OS) at 5 years of $9.8 \%(6.4-14.0)$ with temozolomide versus $1.9 \%$ (0.6-4.4) with radiotherapy alone (HR 0.6, 95\% CI 0.5-0.7; $P \leq 0.0001$ ) [3]. In this study, the O6-methylguanine-DNA methyltransferase (MGMT) promoter methylation status was assessed, and the authors found a considerable rate of disease control in patients with methylated MGMT [3].

Despite the aggressive first-line therapy, consisting of surgery, radiation therapy and adjuvant chemotherapy, the tumors invariably recur and the median survival is 9-12 months.

Second debulking surgery alone does not seem, however, to improve the prognosis, as almost all patients die within 6 months from the second surgery. There are no clearly established chemotherapy regimens for the treatment of recurrent GBM. In this setting chemotherapy strategies in patients with recurrent GBM have used platinum, procarbazine, enzastaurin (alone or in combination with carboplatin) and various combinations. The response rates reported are up to $20-30 \%$ with short progression-free intervals and a median survival rarely exceeding 6 months. In general studies about pharmacological treatment of recurrent GBM have given controversial results [4].

Presently, the new frontier of the pharmacological treatment of GBM is represented by target-based therapies. In fact, on the basis of the encouraging data from the phase-II BRAIN/NSC trial using anti-angiogenic therapy it is currently not known whether biological agents are noninferior to cytotoxic drugs [5].

On the other hand, on the side of cytotoxic drugs recent phase-II trials have demonstrated activity of nitrosoureas 
such as fotemustine in recurrent GBM [6-9]. Fotemustine is a third generation chloroethylnitrosourea containing a phosphoalanine carrier group grafted to the nitrosourea radical. Fotemustine is able to cross the blood-brain barrier; both in vitro and in vivo studies have shown that fotemustine has a marked anti-neoplastic activity on human GBM and medulloblastoma cell lines [10].

The available clinical trials analyzed the efficacy and the safety of fotemustine as single agent in the treatment of 160 patients with recurrent GBM. The treatment was active, with a promising disease control rate, and with a favorable safety profile. These data are interesting if we consider that the majority of the patients had a poor performance status with a compromised hematological status (Table 1). The results showed that the toxicity incidence rate of fotemustine has a peak in correspondence of the induction completion and, it increases with the number of completed cycles [6]. In the trial by Brandes et al. patients with progressive GBM after radiotherapy plus concomitant and/or adjuvant temozolomide received 3-weekly doses $\left(100-75 \mathrm{mg} \mathrm{m}^{2}\right)$ of fotemustine followed, after a 5-week rest, by fotemustine $\left(100 \mathrm{mg} \mathrm{m}^{2}\right)$ every 3 weeks. Forty-three patients were enrolled. Progression-free survival at 6 months was $20.9 \%$; three patients $(7.1 \%)$ had partial response (PR) and 15 (34.9\%) a disease stabilization (SD). The median survival was 6 months (95\% CI 5-7) [7]. Interestingly, disease control rate was significantly greater in patients who started fotemustine at least 3 months after temozolamide administration had been concluded ( 76.9 vs. $26.7 \%, P=0.002$ ). Therefore, the previous experiences, developed mostly in France, had received confirmation from these new trials.

MMGT methylation status is a relevant issue also in the case of nitrosureas, such as CCNU and fotemustine, but at the moment it is still an under-investigated issue in the case of fotemustine. The clinical trial by Fabi et al. did not suggest any correlation between MMGT methylation status and response [6]. However, this study enrolled also anaplastic tumors and the analysis of MMGT promoter was performed in less that $50 \%$ of cases. On the other hand, in the trial by Brandes et al. the methylation status of MMGT was predictive of response $(P=0.044)$ [7]. Therefore, the exiguous number of samples analyzed needs a confirmation in a larger series particularly because the intra-tumoral methylation

Table 1 Phase-II studies with fotemustine as single agent for the treatment of recurrent GBM

\begin{tabular}{lllll}
\hline References & Drug & No. of patients & RR (\%) & ODC (\%) \\
\hline Brandes et al. [7] & FTM & 43 & 7.1 & 42 \\
Fabrini et al. [8] & FTM & 50 & 18 & 62 \\
Scoccianti et al. [9] & FTM & 27 & 29.6 & 48 \\
Fabi et al. [6] & FTM & 40 & 20 & 47.5 \\
\hline
\end{tabular}

$O D C$ overall disease control status may be a dynamic phenomenon dependent on the previous cytotoxic regimen the patient has received [11].

In order to improve the anti-tumor activity of fotemustine in GBM, new combinations between fotemustine and other chemotherapy agents should be explored. In this view, a combination of dacarbazine and fotemustine was used for newly diagnosed GBM with a median TTP from the start of therapy of 9.5 months and a 6-month progression-free survival of 54\% [12].

Fotemustine was also used as salvage chemotherapy with procarbazine in the treatment of temozolomide-treated recurrent glioblastoma patients [13]. In this trial, 54 patients with recurrent GBM were enrolled. Procarbazine was administered as an oral dosage of $450 \mathrm{mg}$ on days $1-2$ and a total dose of $300 \mathrm{mg}$ on day 3. Fotemustine was administered on day $3,3 \mathrm{~h}$ after the last procarbazine intake at a dose of $110 \mathrm{mg} / \mathrm{m}^{2}$. The treatment was repeated every 5 weeks. Six patients $(11.2 \%)$ experienced a neuroradiographic PR and 29 patients (53.7\%) had SD. The median survival time calculated from the first diagnosis was 20.8 months (95\% CI 16.7-24.8).

Another possibility is the combination of fotemustine with targeted therapy agents. The latter, with the exception of bevacizumab in combination with irinotecan [14], gave deluding results in the first phase-II clinical trials in GBM, also for the absence of combination with active cytotoxic chemotherapy. In fact, phase I and II trials with antiangiogenic as AZD2171 (cediranib) or EGFR tyrosine kinase inhibitors (e.g. erlotinib), Akt inhibitors, or PKC inhibitors (e.g. enzastaurin) are presently available. The following considerations should be made in order to have a solid biological rationale for using such targeted therapies in combination with fotemustine. Importantly, the selection of targeted therapies may potentially be dependent on whether the GBM is primary or secondary (transformed GBM), since the biology of these two subclasses seem to be different. For example, EGFR and Akt are particularly relevant in primary GBM given that respectively $\sim 50 \%$ of such tumors harbor either an EGFR amplification, or express EGFRvIII, and that the PTEN-/- genotype is frequently present within these tumors [15]. Furthermore, the inhibition of EGFR (and/or Akt) could potentially ameliorate DNA-repair enzyme activity via DNA-PK [16] and also possibly sensitize cells to apoptosis when combined with cytotoxic agents. For secondary GBM, inhibition of hypoxia-inducible factor (HIF)- $1 \alpha$, may be particularly relevant since many of these tumors harbor isocitrate dehydrogenase-1 mutations $[17,18]$ which prevent the formation of isocitrate to $\alpha$-ketoglutarate which is an essential co-factor for prolyl hydroxylase (PHD) activity, which in turn is essential for HIF- $1 \alpha$ degradation [19]. Moreover, trials including these combinations should also take in account drug interactions with corticosteroids and/ 


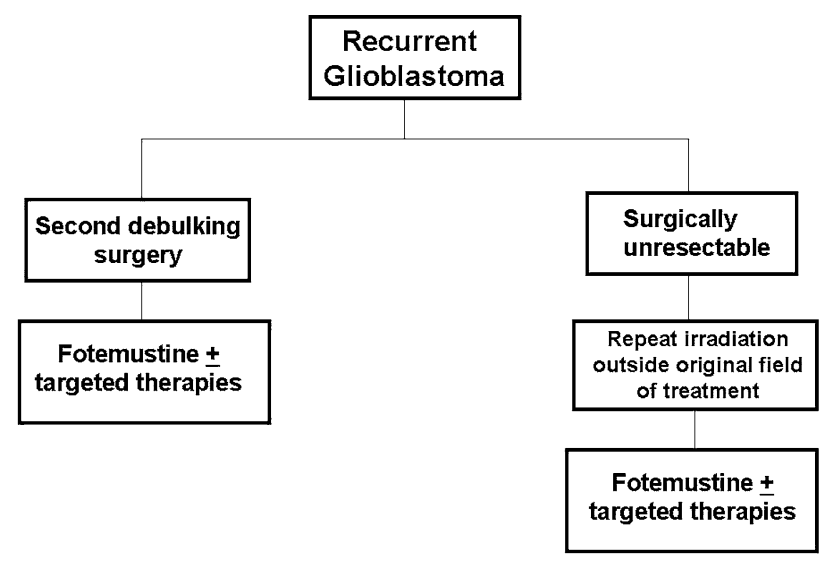

Fig. 1 Algorithm of therapeutic options for recurrent GBM

or anti-convulsants with targeted therapies potentially increasing the toxicities of any future fotemustine \pm targeted therapy regimens. In fact, recurrent GBM patients are frequently given steroids and/or anti-convulsants to control neurological symptoms. In this regard, erlotinib, that is metabolized by CYP3A, has been administered in GBM patients who do not receive EIAEDs in a phase-II clinical trial [20]. Finally, in recurrent GBM, since most disease relapses arise outside the previously irradiated field treated during first-line treatment with the Stupp regimen, reirradiation can be offered to selected patients using conformal planning (IMRT) outside the originally treated area.

Another attractive scenario is the use of fotemustine in polychemotherapy schedules in the neo-adjuvant treatment of GBM. In this view fotemustine (F) $\left(100 \mathrm{mg} / \mathrm{m}^{2}\right.$ day 1$) /$ cisplatin (CDDP) $\left(33 \mathrm{mg} / \mathrm{m}^{2}\right.$ days $\left.1-3\right) /$ etoposide (VP16) $\left(75 \mathrm{mg} / \mathrm{m}^{2}\right.$ days $\left.1-3\right)$ monthly regimen was assessed for efficacy in non-removable glioblastomas at presentation. Interestingly, 7/33 (4/7 alive) were long-term survivors (range 19-67 months) [21].

These results warrant new randomized phase-II trials aimed to optimize treatments based on the combinations between fotemustine and (1) other chemotherapy agents or (2) target-based agents or (3) radiotherapy (see also Fig. 1). These trials should be designed on the basis of biological rationales and should explore possible synergisms above all with targeted-based agents.

We strongly suggest to design large-randomized study to elucidate the role of this old drug as new standard option for the treatment of recurrent GBM.

\section{References}

1. Huncharek M, Muscat J (1998) Treatment of recurrent high grade astrocytoma: results of a systematic review of 1415 patients. Anticancer Res 18(2B):1303-1311
2. Stupp R, Mason WP, van den Bent MJ et al (2005) Radiotherapy plus concomitant and adjuvant temozolomide for glioblastoma. N Engl J Med 352(10):987-996

3. Stupp R, Hegi ME, Mason WP et al (2009) Effects of radiotherapy with concomitant and adjuvant temozolomide versus radiotherapy alone on survival in glioblastoma in a randomised phase III study: 5-year analysis of the EORTC-NCIC trial. Lancet Oncol 10(5):459-466

4. Stupp R, Hegi M E, van den Bent et al (2006) Changing paradigms: an update on the multidisciplinary management of malignant glioma. Oncologist 11:165-180

5. Vredenburgh JJ, Desjardins A, Herndon JE II et al (2007) Bevacizumab plus irinotecan in recurrent glioblastoma multiforme. J Clin Oncol 25(30):4722-4729

6. Fabi A, Metro G, Russillo M, Vidiri A et al (2009) Treatment of recurrent malignant gliomas with fotemustine monotherapy: impact of dose and correlation with MGMT promoter methylation. BMC Cancer 9:101

7. Brandes AA, Tosoni A, Franceschi E et al (2009) Fotemustine as second-line treatment for recurrent or progressive glioblastoma after concomitant and/or adjuvant temozolomide: a phase II trial of Gruppo Italiano Cooperativo di NeuroOncologia (GICNO). Cancer Chemother Pharmacol (Epub ahead of print)

8. Fabrini MG, Silvano G, Lolli I, Perrone F, Marsella A, Scotti V, Cionini L (2009) A multi-institutional phase II study on secondline fotemustine chemotherapy in recurrent glioblastoma. J Neurooncol 92(1):79-86

9. Scoccianti S, Detti B, Sardaro A et al (2008) Second-line chemotherapy with fotemustine in temozolomide-pretreated patients with relapsing glioblastoma: a single institution experience. Anticancer Drugs 19(6):613-620

10. Fischel JL, Formento P, Etienne MC, Gioanni J, Frenay M, DeloVre P, Bizzari JP, Milano G (1990) In vitro chemosensitivity testing of fotemustine (S 10036), a new antitumor nitrosourea. Cancer Chemother Pharmacol 25:337-341

11. Parkinson JF, Wheeler HR, Clarkson A et al (2008) Variation of $\mathrm{O}(6)$-methylguanine-DNA methyltransferase (MGMT) promoter methylation in serial samples in glioblastoma. J Neurooncol 87(1):71-78

12. Fazeny-Dörner B, Veitl M, Wenzel C et al (2003) Survival with dacarbazine and fotemustine in newly diagnosed glioblastoma multiforme. Br J Cancer 88(4):496-501

13. Silvani A, Lamperti E, Gaviani P et al (2008) Salvage chemotherapy with procarbazine and fotemustine combination in the treatment of temozolomide treated recurrent glioblastoma patients. J Neurooncol 87(2):143-151

14. Kreisl TN, Kim L, Moore K et al (2009) Phase II trial of singleagent bevacizumab followed by bevacizumab plus irinotecan at tumor progression in recurrent glioblastoma. J Clin Oncol 27(5):740-745

15. Cancer Genome Atlas Research Network (2008) Comprehensive genomic characterization defines human glioblastoma genes and core pathways. Cancer Genome Atlas Research Network. Nature 455(7216): 1061-1068

16. Mukherjee B, McEllin B, Camacho CV et al (2009) EGFRvIII and DNA double-strand break repair: a molecular mechanism for radioresistance in glioblastoma. Cancer Res 69(10):42524259

17. Parsons DW, Jones S, Zhang X et al (2008) An integrated genomic analysis of human glioblastoma multiforme. Science 321(5897):1807-1812

18. Yan H, Parsons DW, Jin G et al (2009) IDH1 and IDH2 mutations in gliomas. N Engl J Med 360(8):765-773 
19. Zhao S, Lin $\mathrm{Y}, \mathrm{Xu}$ W et al (2009) Glioma-derived mutations in IDH1 dominantly inhibit IDH1 catalytic activity and induce HIF1 alpha. Science 324(5924):192-194

20. Raizer JJ, Abrey LE, Wen P et al (2004) A phase II trial of erlotinib (OSI-774) in patients with recurrent malignant glioma not on EIAEDs. J Clin Oncol 22:107s
21. Frenay M, Lebrun C, Lonjon M et al (2000) Up-front chemotherapy with fotemustine (F)/cisplatin (CDDP)/etoposide (VP16) regimen in the treatment of 33 non-removable glioblastomas. Eur J Cancer 36(8):1026-1031 